\title{
INVESTIGACIÓN INTERDISCIPLINARIA Y TRANSDISCIPLINARIA COMO TENDENCIA EMERGENTE DE LO SISTÉMICO COMPLEJO DESDE EL PENSAMIENTO CRÍTICO
}
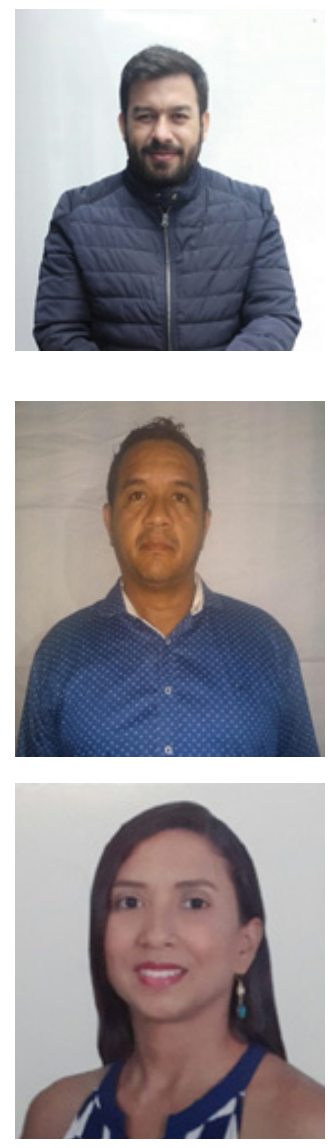

Fecha de recepción: 20/03/2019

\author{
Daniel Alejandro González Ortiz \\ IE del Carare CICA, Cimitarra Santander, Colombia \\ danielboone1734@gmail.com
}

\section{Luis Alfonso Padilla Doria}

IE German Vargas Cantillo, Cereté Córdoba

Sekama2013@gmail.com

\section{Nelly María Zúñiga Díaz}

IE Santa Rosa de Lima, Montería Córdoba

nemazudi@gmail.com

\section{RESUMEN}

En el presente escrito se hace un recorrido por el conocimiento, cómo este se genera y cómo puede seguir cambiando y enriqueciéndose, es un proceso en continua transformación, por eso es necesario que se hable de la complejidad de todo este sistema cognoscitivo. El conocimiento es algo que debe servir para el mejoramiento de la sociedad, el bien de todos, pero es tan complejo entender todo, que se requiere mirarlo desde diferentes vistas, por eso la importancia de observarlo desde varias disciplinas, que ayuden y aporten aumentar el conocimiento en pro del beneficio de la humanidad; es así como nace la interdisciplinariedad, para poder ver objetivamente los problemas y poder dar soluciones efectivas a las dificultades que se presenten. También surge la transdisciplinariedad, la cual: "es una forma específica de interdisciplinariedad en la cual, los límites entre y más allá de las disciplinas se trascienden y el conocimiento y las perspectivas desde 
diferentes disciplinas científicas, así como fuentes no científicas son integradas" (University of Southampton, 2005, Citado por Henao C., 2017, p. 184). Al final del ensayo se hace un análisis del porque las universidades deben buscar modelos propios de enseñanza - aprendizaje basados en grupos interdisciplinarios que conlleven a una transdisciplinariedad en la educación. Como dice Sánchez J.R. (2019, p.82) "hoy en día la transdisciplinariedad se asoma como una alternativa que parece avanzar hacia una verdadera integración del saber humano".

Palabras Clave: Interdisciplinariedad, Transdisciplinariedad, Complejidad.

\title{
INTERDISCIPLINARY AND TRANSDISCIPLINARY RESEARCH AS AN EMERGING TREND OF THE COMPLEX SYSTEM SEEN FROM THE DEVELOPMENT OF CRITICAL THOUGHT
}

\begin{abstract}
In this paper, a tour of knowledge is made, how it is generated and how it can continue to change and enrich itself, it is a process in continuous transformation, so it is necessary to talk about the complexity of this entire cognitive system. Knowledge is something that should serve to improve society, the good of all, but it is so complex to understand everything, it is necessary to look at it from different views, so the importance of observing it from various disciplines, that help and contribute to increase the knowledge for the benefit of humanity; This is how interdisciplinarity is born, in order to objectively see the problems and be able to provide effective solutions to the difficulties that arise. Transdisciplinarity also arises, which: "is a specific form of interdisciplinarity in which, the boundaries between and beyond the disciplines are transcended and knowledge and perspectives from different scientific disciplines, as well as non-scientific sources are integrated" (University of Southampton, 2005). Cited by (Henao C., 2017). At the end of the essay an analysis is made of why universities should look for their own teaching - learning models based on interdisciplinary groups that lead to transdisciplinarity in education. As Sánchez J.R. (2019) "Today, transdisciplinarity is seen as an alternative that seems to move towards a true integration of human knowledge."
\end{abstract}

Keywords: Interdisciplinarity, Transdisciplinarity, Complexity. 


\section{INTRODUCCIÓN}

Estamos en un mundo y en una era globalizada, los avances tecnológicos en todos los campos del conocimiento han hecho que la comunicación entre Instituciones educativas, comunidades de aprendizaje, grupos de investigación, universidades, sea sencillo y fácil de realizar, algo que fluye con mucha naturalidad y aparta las barreras que existieron por la falta del mismo. A raíz de todas estas comunicaciones espontaneas entre las comunidades del pensamiento, se ha hecho fácil la interacción entre varias disciplinas y a la luz de un problema se puede abarcar y dar solución efectiva a éste, viendo las causas y las soluciones desde diferentes ángulos o puntos de vista, para luego escoger entre todos, la solución más óptima en pro del beneficio común.

Si bien es cierto, aun se conoce de corrientes pedagógicas que se niegan a los cambios, encontrando universidades e Instituciones educativas que continúan enseñando de forma mono disciplinar y además el alcance de sus investigaciones, de sus egresados se queda en lo local, regional o nacional. Las universidades deben apuntar a una educación globalizada e internacional, que su alcance no sea su campus universitario, sino el mundo entero.

La misma globalización e internacionalización de la educación que se impone de manera avasallante a la sociedad nos ha llevado a dar un realce e importancia al estudio de lo interdisciplinario pues esta nace de la misma necesidad de oponerse al encierro de lo disciplinario, en otro tiempo algunas escuelas de pensamiento fueron marginadas por proyectarse a lo interdisciplinario, pero hoy día son muy honradas y tomadas como paradigmas para lo que se entiende modernamente por universidad. Por eso es que se necesita que las universidades no se estanquen y sigan visionando y avanzando a las necesidades y cambios que se generan en la sociedad.

Teniendo en cuenta las proyecciones y la visión que deben tener las universidades es que se crea la necesidad de reformar el pensamiento y, al mismo tiempo, las instituciones educativas, con el fin de promover una educación acorde con las necesidades de la sociedad contemporánea y con egresados involucrados con los problemas de la sociedad, con una mentalidad completamente crítica, madura antes los cambios paradigmáticos, con perspectiva global, con algunos procesos de complejización y policompetente como investigador.

La interdisciplinaridad y transdisciplinaridad son dos vertientes que juntas configuran una tendencia que tiene su arraigo en lo paradigmático complejo, pues irrumpe en el paisaje científico contemporáneo provocando sin lugar a dudas un replanteo de los fundamentos mismos de la racionalidad imperante, repercutiendo positivamente en la pedagogía dado que presta mucha 
atención a los conceptos que se van desarrollando en las ciencias, cuestionando sus fundamentos epistémicos.

El pensamiento complejo ha cuestionado no sólo el concepto mismo de ciencia, sino que ha anunciado que lo que ha cambiado es la naturaleza misma de lo que entendemos por conocimiento. No obstante, se verían también afectados los procesos de construcción de saberes y aprendizajes que ocurren en el marco de las mediaciones pedagógicas.

Para que funcione toda esta parafernalia hay que comenzar apuntando al desarrollo de un pensamiento crítico dentro de la actividad pedagógica en todos los ámbitos ya sea escolar o universitario porque de esta manera se da viabilidad a todos los cambios que están dándose en el marco del desarrollo transversal desde lo inter y transdisciplinario. Aunque a veces surgen preguntas como, por ejemplo: al hablar de lo transdisciplinario nos podemos plantear. ¿Es posible tener una planeación y reflexión sobre contenidos transversales en educación, sin una aproximación transdisciplinaria sobre la complejidad de lo real?

La presente investigación ha sido un trabajo que centra su estudio o investigación metodológica desde un paradigma cualitativo con enfoque hermenéutico, mediante un análisis documental como tipo de estudio; Este proceso, requiere de la aplicación de estrategias especializadas en la búsqueda, selección, organización y análisis de un conjunto de producciones escritas, que den respuesta a una o varias preguntas sobre un tema (Bermeo, Hernández, y Tobón, 2017, p.105). El análisis bibliográfico se hará de diversas publicaciones recientes tanto nacionales como internacionales en los temas relacionados, como son conocidos la interdisciplinaria y transdiciplinaria en el proceso de enseñanza - aprendizaje de doble vía, en diferentes áreas de formación e investigación, para así proponer, aplicar, desarrollar y conocer el conocimiento científico en la formación continua de niños, jóvenes y adultos en diversos programas de educación, desde primaria, secundaria, y superior.

En el presente ensayo se hará un recorrido que comienza en la adquisición del conocimiento, de las competencias, luego se pasará a una mirada por el pensamiento, sistema o paradigma complejo base de la interdisciplinariedad y transdisciplinariedad, para al final analizar estas disciplinas en la generación del conocimiento desde las Instituciones Educativas y las Universidades. 


\section{INVESTIGACIÓN INTERDISCIPLINARIA Y TRANSDISCIPLINARIA COMO TENDENCIA EMERGENTE DE LO SISTÉMICO COMPLEJO VISTO DESDE EL DESARROLLO DEL PENSAMIENTO CRÍTICO}

Se presume que la sociedad actual tendrá importantes y acelerados cambios en un tiempo no mayor de 50 años, incluso éstos cambios podrán ser más significativos e importantes que los surgidos en el último siglo. Convivimos en una sociedad cambiante y revolucionaria ante el desarrollo del mundo en sus diversas áreas del conocimiento y la generación del mismo. El desarrollo económico, las políticas gubernamentales, la cultura, el arte, la ciencia, el desarrollo social y comunitario junto con el conocimiento se involucra en las diferentes fases de los desarrollos académicos, pedagógicos, siendo sobresaliente en lo educativo. La sociedad es ligera en el desarrollo psicopedagógico, en los procesos de enseñanza-aprendizaje, en la evolución o cambios procesados en respuesta al mismo.

Martínez y Sánchez (2018, p. 64) citan:

"Las instituciones educativas deben de forjar una combinación de conocimientos, capacidades y actitudes en el estudiante que se encuentra en un contexto determinado; esto con el fin de su realización, desarrollo personal, inclusión social y preparación al mundo laboral" (Gómez, Miralles y Molina, 2015). Así mismo en el contexto mundial la visión integral de las competencias es una aportación por parte del profesionista al integrar los valores, la cultura y la ética, que permitan formar criterios que contribuyan a la aplicación de sus habilidades y conocimientos de manera responsable contribuyendo a un bien común (Ortiz, Vicedo y García, 2016).

Sin embargo, son las mismas competencias las que se convierten en lo verdaderamente importante de cualquier sistema educativo, de cualquier país. Pues las competencias, resaltan o ven la importancia de los individuos en cuanto al desarrollo y aplicación de habilidades, destrezas, didáctica y otras, que hacen del individuo, que puedan generar respuestas ante lo cambiante, ante lo dinámico, respondiendo con adaptabilidad, formulación y solución de problemas con respuestas acertadas.

Se puede mencionar que a través del desarrollo de competencias se genera el conocimiento (como se muestra en la Gráfica 1), como respuesta a un proceso educativo por un sistema, en un mundo globalizado. Es la misma obtención del conocimiento, la cual ha impuesto exigencias y retos que involucran cambios permanentes en los desarrollos educativos y sus autores o estudiantes en la dinámica educativa de formación de profesionales, licenciados, técnicos y otros con grados 
académicos que buscan continuamente respuestas en medio de los paradigmas y los ambientes reales de aprendizaje.

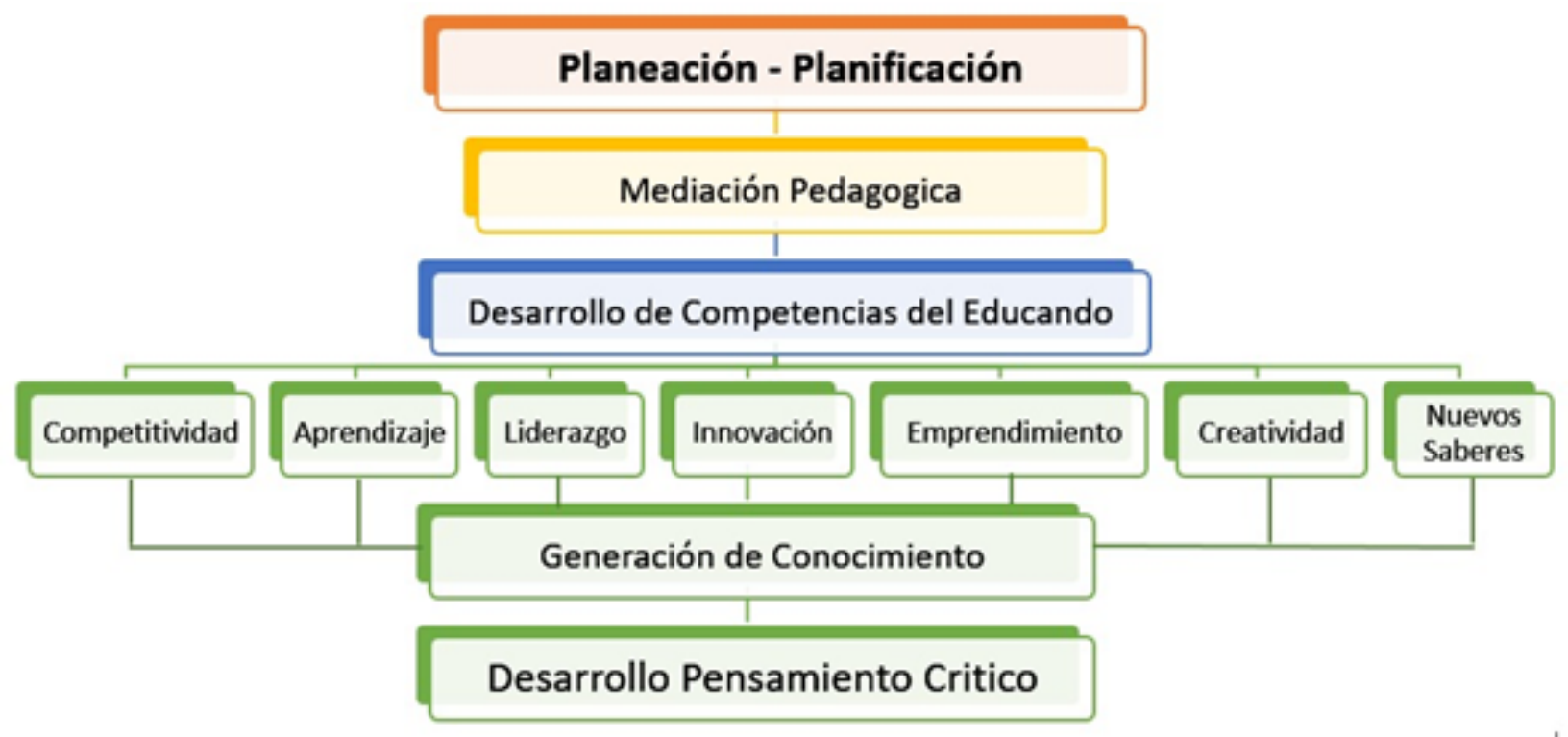

\section{Gráfica No. 1. Mapa mental del desarrollo de las competencias y generación de conocimiento con pensamiento crítico. Elaborado por los autores.}

El conocimiento es el producto de la imaginación realizada, basada en hechos teóricos como conceptuales que son adquiridos y practicados por los individuos, manifestado en la inteligencia. Si bien es cierto, el conocimiento se ha convertido en lo fundamental para el desarrollo social y económico de las comunidades. Como lo menciona Romero, "este ha adquirido un nuevo valor de producción a nivel industrial, ya que a partir de él se llevan a cabo acciones derivadas de la combinación de la información, experiencias, valores y normas internas" (Romero, 2007, citado por citado por Martínez y Sánchez, 2018, p. 65).

Es tan amplio y complejo entender cómo se genera el conocimiento que Lodge (2018, citado Ferreira, Altamirano, López y González, 2019, p. 36) expone: "La educación es un fenómeno complejo, multidimensional, contextual y social; por lo tanto, intentar comprender cómo se produce únicamente a través de una única perspectiva es insuficiente, e incluso problemático".

Tener el conocimiento es dominar incluso las naciones. Hoy día, el conocimiento se desarrolla por cualquier parte, no es solo de las instituciones académicas o universidades son las responsables del mismo. En la época actual, época real, no solo el conocimiento lo domina las grandes y prestigiosas universidades público - privadas. La sociedad reclama personas con 
alto contenidos de criticidad, curriculum, responsabilidad, coherencia, exigencia, dinamismo, pero sobre todo mayor adquisición académica, representada en títulos, para así afrontar los retos que generan cambios en el proceso educativo como en las personas. El conocimiento es el saber adquirido de una experiencia o momento significativo. Hace referencia también a la inteligencia como la razón causal de las personas que logran producir cambios, conceptos, teorías, leyes y otros, en una sociedad que debe formular, aplicar y desarrollar conocimientos, que deberá manipularse en la búsqueda de soluciones frente a la presentación de dificultades y otros.

El paradigma sobre la investigación interdisciplinaria y transdiciplinaria como ejes principales del conocimiento y los sistemas educativos en procesos transversales con la educación, se traduce en la búsqueda del aprendizaje significativo como principal garante de la construcción de conceptos teóricos-prácticos y por ende la construcción y adquisición del conocimiento en un proceso llamado de enseñanza-aprendizaje en doble vía. No se establece con esto, que sólo importe la formación de la dimensión cognoscitiva de niños y jóvenes, por el contrario, los fines señalados para la educación recalcan su carácter integral y pluridimensional, siendo éste mejor desarrollado en un marco estratégico de posibilidades, responsabilidades, donde sus sujetos y agentes son plenamente conscientes de los objetivos que se han trazado y están en su búsqueda desarrollando un mejoramiento continuo de cada uno de los procesos con eficacia y eficiencia, que trabaja cada día para la obtención de sus logros.

Es claro que el aprendizaje significativo se produce paso a paso, y por tanto corresponde a un proceso en el que se desarrollan las potencialidades de los escolares en sus entornos propios. Estas potencialidades son de diferentes clases y en el campo cognitivo en particular, corresponden a competencias interpretativas, argumentativas y propositivas, que, en la educación, son objeto de evaluación para pruebas externas y así evaluar la calidad de conocimiento adquirido en el desarrollo de competencias por cada individuo e instituciones consagradas a la formación integral de personas en los diferentes niveles educativos de la vida para cada educando.

La generación del nuevo conocimiento, visto desde la dinámica global, con visión sistémica en la formación integral de las personas en un mundo acelerado, globalizado, donde los cambios se han convertido en una evolución continua que no se va detener, por el contrario, será cada vez más rigurosa, exigente y dinámica. Es de obligatoriedad generar un nuevo conocimiento con altos índices de criticidad, donde se reflexione las diversas problemáticas generando contextos de análisis detallado sobre la información manipulada, los entornos naturales, tecnológicos y el uso de las TICS que hacen que el mundo esté en continua comunicación. Lo que nos obliga a responder a situaciones diarias, cada vez con mayor complejidad, bien sea por resultados en el andar académico 
o surgimiento de problemáticas en su avance educativo y de la producción del nuevo conocimiento.

Cuando hablamos de investigación interdisciplinaria y transdisciplinaria, no podemos eludir el concepto de complejidad, que emerge de lo sistémico, es decir para ahondar en esta tendencia tenemos que hacer un análisis minucioso de los principios que regulan los sistemas complejos. Esto ha incursionado en muchas ramas del saber, pero con mucho mas vigor en las ciencias sociales, en la actualidad va ganando bastante terreno en la medicina y las ciencias físicas, en esta última muestra resultados sorprendentes e insospechados, entonces lo que vemos es una tendencia que permite grandes avances a medida que se desarrollan la tecnología y la informatización de las teorías científicas.

En forma general hay variados y múltiples ejemplos de sistemas complejos, como es el caso del funcionamiento total del cerebro, problema de la contaminación en las ciudades, los ecosistemas en proceso de desequilibrio, el problema de tres cuerpos en movimiento, que es un problema clásico de la Física, en donde se describe el comportamiento de dichos cuerpos en interacción y para lo cual, no se tiene en la actualidad una solución analítica.

La época actual se enmarca en una sociedad neoliberal, globalizada y postmoderna, la complejidad de los nuevos modelos y teorías científicas nos está obligando considerar la información como un insumo de inmenso valor. La revolución que estamos viendo es decir la científico-tecnológica está dando origen a unos cambios profundos y trascendentales en la sociedad moderna, básicamente cambios en las mentalidades, perspectivas y valores humanos.

En este contexto paradigmático asumimos una dicotomía “dato-información”, entendiéndose el primero como el objetivo y lo segundo como algo subjetivo. Por el contrario, si abordamos lo sistémico desde el paradigma de la complejidad inmediatamente se omite esta falsa dicotomía y más bien se acentúan elementos comunes de al menos, cuatro aspectos, estos son: la información subjetiva, la información objetiva, el proceso empírico de sensaciones-percepciones y las acciones sobre la información, consideradas dentro de un complejo dialéctico.

Los seres humanos hemos logrado interactuar de una manera eficiente, y cada vez más inteligente, con nuestro entorno, esto nos ha llevado aumentar la capacidad de reflejar, de manera creativa, la realidad por medio de cálculos matemáticos y modelos formales. Podemos decir que los nuevos procesamientos de información provocaron la aparición de lenguajes que de una manera u otra organizaron la mente de quien los utilizó, cambiando drásticamente, nuestro modo y estilo de vida. 
En este proceso de reconstrucción humana, el conocimiento adquirió una nueva dimensión y, al transformarse en poder, nos definió un nuevo cuadro ontológico que se basó en la tríada: información-energía-materia. Para poder comprender este entramado conceptual de lo sistémico complejo, es importante entender la problemática existente entre complejidad e interdisciplina, pues con estas dos vertientes va emergiendo lo que podíamos llamar el paradigma de la complejidad con proyecciones epistémicas, éticas y políticas. Todo esto puede originar distintas vertientes teóricas, metodológicas y epistemológicas nutriendo un campo paradigmático emergente, producto de las distintas vertientes que convergen y a la vez divergen y que en cierta forma coadyuvan para que se dé un desarrollo integral de un paradigma de la complejidad.

En todo este andamiaje surge un conflicto conceptual debido a que las diversas vertientes de la complejidad no son equivalentes en sus planteamientos conceptuales ni tampoco necesariamente compatibles en sus propuestas metodológicas, la razón de esto es clara; toda construcción teórica se asienta en supuestos de orden epistemológico, ontológico y axiológico que es realmente necesario explicitar y analizar con todo vigor.

Lo dicho anteriormente trae serias implicaciones, debido a que las clásicas fronteras disciplinarias y profesionales se han visto cuestionadas en su capacidad de ofrecer respuestas a los crecientes problemas multidimensionales, globales y complejos que enfrentamos. Las investigaciones científicas innovativas, el resurgimiento de nuevos campos híbridos profesionales y disciplinares, la colaboración inter y transdisciplinar, todo este tipo de situaciones de aprendizaje en búsqueda de la comprensión y la solución de problemas, la educación desde lo local, entre otras cosas, comenzaron a evidenciar, con sus resultados, la necesidad de impulsar modelos más complejos y colaborativos en la construcción del conocimiento. A este, relativo, reciente procedimiento en la producción del conocimiento se le ha llamado de distintas maneras, entre ellas, paradigma de la complejidad.

Es importante dejar claro el concepto de paradigma. Para ser más asertivos adscribiremos la siguiente y sencilla definición de Morin: un paradigma es "la relación lógica entre conceptos matrices que comandan todas las teorías y todos los discursos que de ella dependen" (Morin, 2016, p.39). Esta definición nos resulta muy útil y puede ser aplicable a todas las vertientes de la complejidad.

En efecto, en términos concretos no se ha realizado ningún aporte decisivo a nivel teórico ni metodológico que señale cómo podría efectuarse el vínculo entre el pensamiento complejo y los sistemas complejos [...] según esto queda abierta la posibilidad de un trabajo a futuro que articule 
ambos enfoques (Rodríguez Zoya \& Rodríguez Zoya, 2014, p.126). Por otra parte, el pensamiento complejo y los enfoques holistas deben exigirse mayor rigor en la construcción de sus principios y métodos de conocimiento. Esto puede darse, impulsando la idea de que el pensamiento y el conocimiento complejo deben generar un circuito entre los principios de la ciencia clásica y los principios derivados de las distintas versiones de la complejidad. Al respecto escribe José Luis Solana:

"Para el pensamiento complejo, el análisis, la distinción, la abstracción, la cuantificación, la especialización y la precisión, la lógica clásica, la metodología, los algoritmos y los modelos (operaciones de conocimiento cuya potencia y capacidad de rendimiento nunca deberían olvidarse ni infravalorarse), resultan, deben ser, tan necesarios como la síntesis, la relación, la concretización, la cuantificación, la interdisciplinariedad y las miradas a la totalidad, la dialógica, el método, las metáforas y los discursos. En caso contrario, las operaciones intelectivas complejizadoras se absolutizan y tornan simplificadoras" (Solana, 2011, p.13).

Esto tendría un significado, por ejemplo, que habría que revisar y, en su caso, complementar la teoría de Edgar Morin a la luz de los avances de las ciencias de la complejidad. En este sentido está trabajando el Centre Edgar Morín (París, EHESS / CNRS), buscando acercar y relacionar el pensamiento complejo y las ciencias de la complejidad a través de las posibilidades que ofrece los sistemas adaptativos complejos.

En relación con lo anterior, habría que profundizar en el trabajo de operativización, aplicación e implementación empírica de los principios epistemológicos y estrategias intelectivas que se derivan de diversas vertientes de la complejidad para observar su potencial comprensivo o explicativo en el estudio de realidades concretas. "Parece necesario un trabajo de "operativización" del pensamiento complejo”. (Roggero Pascal, 2013, p. 120).

Otro aspecto necesario para avanzar en la discusión y reflexión sobre la complejidad es confrontar, abiertamente, las diferencias entre diversas posiciones y dialogar sobre ellas. Con estos encuentros no se pretendería llegar, necesariamente, a algunos acuerdos; lo que se buscaría, más bien, sería profundizar en el conocimiento mutuo o entre los enfoques en discusión, comprender las diferencias en su justa dimensión y continuar cuestionándonos con mejores elementos para saber más sobre nuestras potenciales complementariedades o para seguir explorando diversos caminos. Para este propósito, habría que estar alertas para no quedar atrapados, por nuestra propia voluntad y ceguera, en los planteamientos de los fundadores de alguna de las corrientes de pensamiento 
Investigación interdisciplinaria y transdisciplinaria como tendencia emergente de lo sistémico complejo desde el pensamiento crítico

Daniel A. González O.; Luis A. Padilla D.; Nelly M. Zúñiga D. (63:83)

—absolutizándolos y dedicándonos a repetir sus planteamientos, convirtiéndonos en dogmáticos discípulos-. En todo caso, se trataría de regresar a las fuentes de los pensadores fundadores para regenerar y reconstruir su pensamiento a partir de los nuevos avances del conocimiento. De esa manera, se pueden tener bases para buscar integrar la complejidad en una concepción más amplia.

Quizá, las palabras que Álvaro Malaina (2016, p.57) dedica a estas dos vertientes de la complejidad nos puedan servir de síntesis en torno a lo mucho que nos falta dialogar entre estos enfoques:

[...] la ciencia de los sistemas complejos es un proyecto aún en curso, que todavía tiene mucho camino que recorrer, en lo que respecta al grado de sofisticación y adecuación de sus técnicas computacionales para captar en su plenitud la complejidad y sobre todo en la superación del paradigma de simplificación que aún constriñe sus trabajos, convirtiéndolos en modelos cerrados que dejan escapar buena parte de la complejidad sistémica, especialmente, la que tiene que ver con el sujeto observador. Lo cual vuelve necesaria la integración de la ciencia de sistemas complejos en el horizonte visionario más vasto del pensamiento complejo, del cual debería nutrirse de la misma forma que este debiera, por su parte, nutrirse de aquella [...].

Existen dos maneras de entender lo incognoscible para el ser humano, diría Morin, como enigma o como misterio. El enigma es lo desconocido hasta el momento, pero puede conocerse, racionalmente, en el futuro con los recursos científicos y tecnológicos actuales o por desarrollar. En cambio, el misterio son aquellas preguntas que no pueden ser respondidas con la razón, pues remiten al misterio de la existencia, por ejemplo ¿por qué existe el cosmos y no nada? Tanto el pensamiento complejo como los enfoques holistas reconocen los límites del conocimiento empírico-racional para afrontar ciertas cuestiones filosóficos, por decir lo menos. En palabras de Morin: El milagro del conocimiento es conducirnos, más allá del estado de la complejidad, a las fronteras del Misterio. Todo aquello que nosotros hemos aprendido del Universo ha revelado un abismal misterio de la realidad [...] misterio de la vida sobre la tierra, tanto sorprendente desde su nacimiento, y no menos sorprendente en su evolución, misterio de lo humano, misterio de la consciencia. Estamos rodeados en adelante por insondables misterios que se conectan en el gran y supremo Misterio (Morin, 2015, p.84).

Teniendo claro los conceptos de lo sistémico complejo sobre lo cual se edifica la investigación interdisciplinaria, esta debe abordarse desde tres grandes pilares lo epistemológico riguroso, metodológico factible y político crítico (Ver Grafica 2.). Esto implica tomar una posición ideológica 
explícita que involucre una cierta concepción y relación entre ciencia y sociedad: definitivamente el conocimiento interdisciplinario es necesario y conveniente para una política transformadora de los problemas complejos que afectan la vida de los pueblos en Latinoamérica. Lo anteriormente expuesto nos lleva a pensar de manera más aterrizada lo que entendemos por interdisciplinario llevándonos a enunciar tres consideraciones sobre la interdisciplina.

1. Se puede entender como un concepto multidimensional donde se involucran aspectos epistemológicos, metodológicos y cognitivos, dándose condiciones institucionales, culturales y educativas para su desarrollo. Todas estas dimensiones se relacionan mutuamente con carácter político.

2. Puede mirarse como una estrategia metodológica necesaria e imprescindible para estudios complejos de situaciones.

3. Cuando se habla de la política de la interdisciplina hace alusión, por un lado, a esas condiciones necesarias para una práctica interdisciplinaria y, por el otro, a las consecuencias potenciales del trabajo interdisciplinario en el plano científico y social.

"La investigación interdisciplinaria es una colaboración de varias disciplinas, pero en este caso, conceptos, metodologías, o epistemologías son intercambiadas e integradas explícitamente, resultando en un enriquecimiento mutuo" (Flinterman, Teclemariam-Mesbah \& Broerse, 2001, Citado por Solorzano, Henao, Bracho, Arboleda, García, Aguirre, y González, 2017, p,183). Se puede inferir que las disciplinas de estudio tienen afinidad en cuanto a su objeto de estudio se trata. Sin embargo, los esfuerzos en diferentes procesos de investigación por diseñar nuevos enfoques interdisciplinarios son enormes para la búsqueda de la objetividad.

Como se menciona, los proyectos interdisciplinares hacen uso de las inteligencias multiplex en la conformación del conocimiento colaborativo con profesionales o investigadores de diferentes áreas del conocimiento, para así identificar problemáticas con varios enfoques, encaminando esfuerzos en la búsqueda de soluciones, representada el progreso académico desde la perspectiva en los diferentes intereses en cuanto conocimiento se trate. "Un equipo interdisciplinar aspira a un nivel más profundo de colaboración (que un equipo multidisciplinario), en el cual quienes constituyen diferentes acervos combinan su conocimiento mutuamente para completar distintos niveles de intereses planeados" (Bernard-Bonnin, Stachenko, Bonin, Charette \& Rousseau, 1995, Citado por Solorzano et al., 2017 p. 183) 
Investigación interdisciplinaria y transdisciplinaria como tendencia emergente de lo sistémico complejo desde el pensamiento crítico

Daniel A. González O.; Luis A. Padilla D.; Nelly M. Zúñiga D. (63:83)

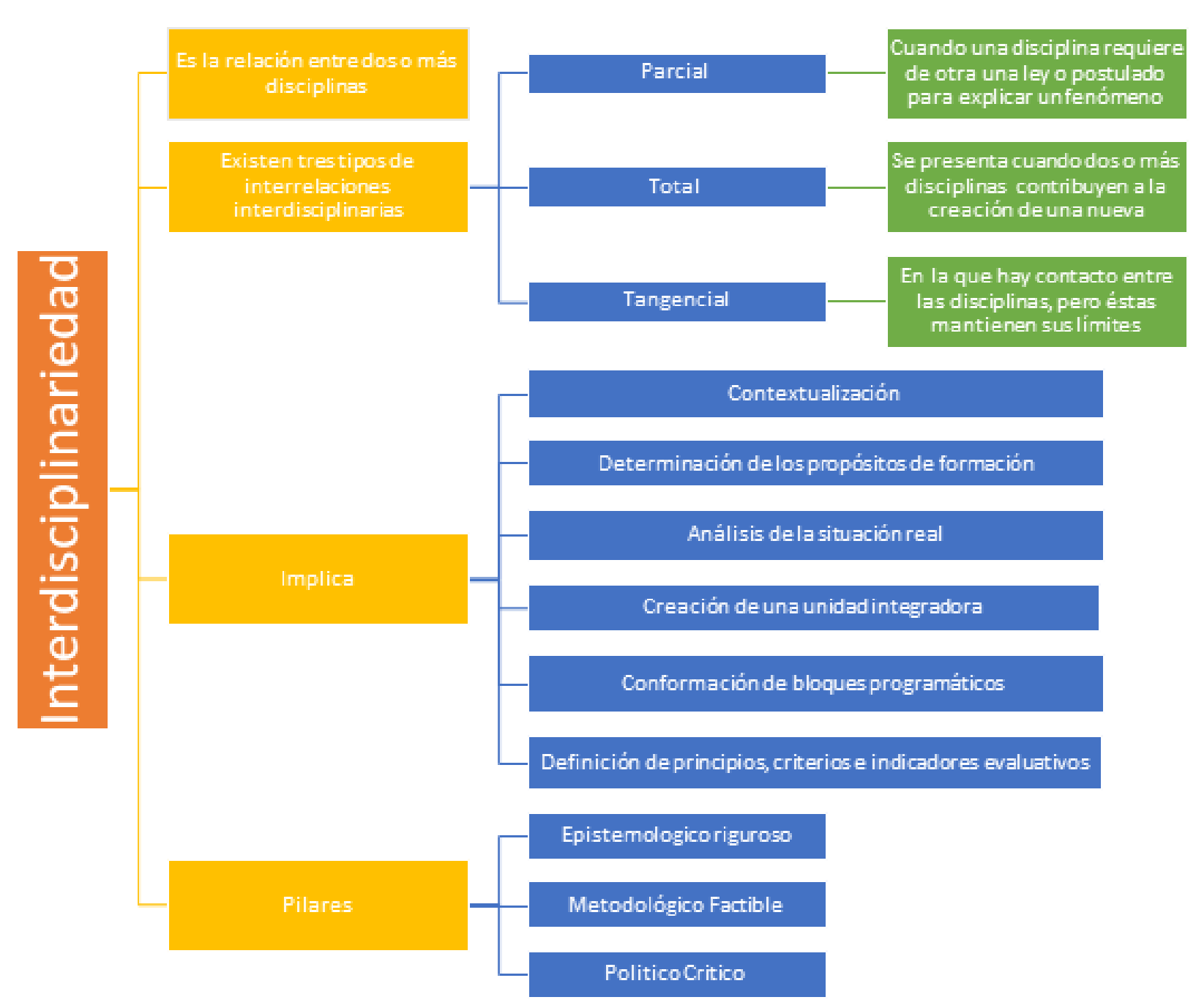

Gráfica 2. Generalidades Interdisciplinariedad. Elaborada por los autores.

Si bien es cierto, la exigencia sobre cualquier proceso académico con visión holística tendrá los mejores resultados en cuanto a formación de los educandos con un nuevo conocimiento basado en el desarrollo del pensamiento crítico, reflexivo, con humanismo en la construcción de personas integrales para una mejor sociedad, amigable con los entornos ambientales desarrollando inteligencias multiplex en pro de mundo globalizado para la creación de nuevas estrategias que hagan nuestra estadía en esto que llamamos planeta más amena, más familiar, que puedan aumentar los procesos altruistas con enfoques interdisciplinarios teniendo en cuanta el humanismo de cada ser.

Mirando lo sistémico complejo desde lo transdisciplinario vemos la dificultad de transformar un sistema que siempre se resiste al voluntarismo tecnopolítico esto se ve patentado en el desacierto en la aplicación de las distintas reformas educativas de países occidentales, ¿qué es lo que pasa? 
Porque no se halla una solución al sistema educacional que responda a las necesidades educativas de un país y/o la región? Los gobiernos de los países no han ahorrado esfuerzos en acortar la brecha educativa a pesar de las diferencias en las posibles soluciones en las formas y caminos elegibles para su aplicación, parten del mismo diagnóstico: los distintos niveles educativos y de formación científicos y tecnológicos, y por los cambios emergentes en el orden social, profesional se encuentran obsoletos por causa de los profundos cambios económico y cultural. Las organizaciones educativas suman a su desorientación actual, un creciente aislamiento institucional con respecto a los desafíos sociales y laborales, en particular, y a las transformaciones de la cultura y la vida en general.

La transdisciplinariedad no se esfuerza por dominar muchas disciplinas, su objetivo es abrir todas las disciplinas a que compartan y que observen más allá de ellas. "es una forma específica de interdisciplinariedad en la cual, los límites entre y más allá de las disciplinas se trascienden y el conocimiento y las perspectivas desde diferentes disciplinas científicas, así como fuentes no científicas son integradas" (University of Southampton, 2005, Citado por Solorzano et all., 2017 p. 184). Los proyectos o investigaciones transdisciplinares, sus investigadores y profesionales se esfuerzan por desarrollar trabajos colectivos en el desarrollo del conocimiento complejo, a partir de trabajos grupales donde estos investigadores se enfocan en un problema en común con el tiempo suficiente que integra los diversos conocimientos de cada uno de sus integrantes formados en diferentes áreas del conocimiento, y cada uno aportara desde cada perspectiva disciplinar por separada.

Es así que la integralidad de los saberes y formaciones académicas en diferentes contextos, son encaminadas a la búsqueda de soluciones claras, oportunas, pero sobre todo apunta a la construcción de nuevos modelos con enfoques diversos sobre el conocimiento, basados en la transdisciplinariedad con alta criticidad en generación del nuevo conocimiento. "El grado de integración entre disciplinas se restringe a los resultados de investigación" (Flinterman, Teclemariam- Mesbah \& Broerse, 2001, Citado por Solorzano et al., 2017, p. 183).

En la gráfica 3 se pueden apreciar algunas generalidades de la transdisciplinariedad, como es el concepto, los pilares y su finalidad. Teniendo en cuenta los pilares es que al comienzo se hizo énfasis en el pensamiento complejo, pues es una de las bases para llegar a la transdisciplinariedad; también en páginas anteriores se habló mucho del conocimiento pues esto solo se adquiere de otro de los pilares como lo es la realidad para poder alcanzar su finalidad que es la comprensión del mundo presente desde múltiples ángulos y vistas, entendiendo de una manera integral los problemas, para poder ser objetivos y brindar mejores propuestas y soluciones a las problemáticas presentadas en este mundo cambiante con una formación humanista. 


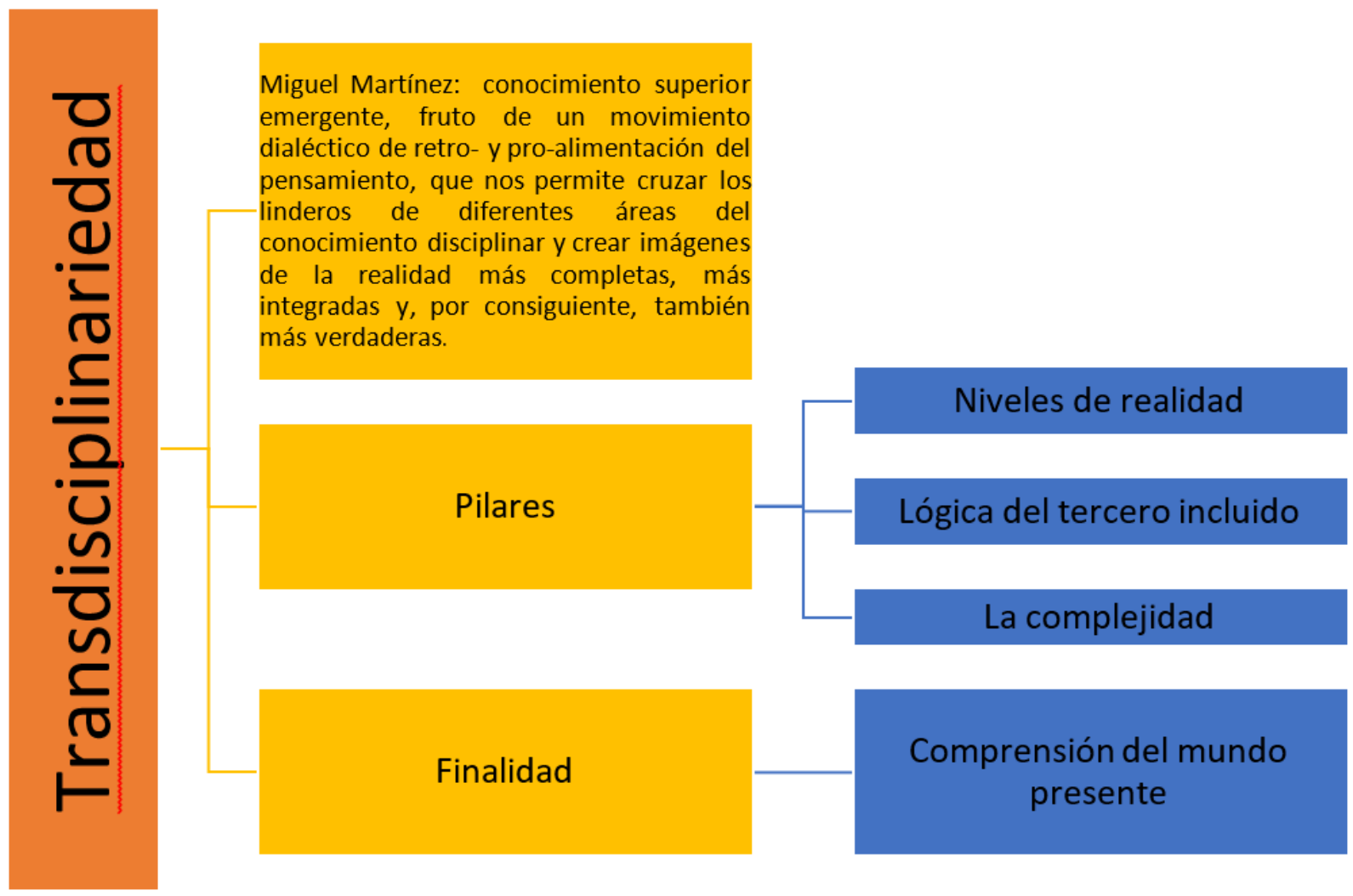

\section{Gráfica 3. Generalidades de la Transdisciplinariedad. Elaborada por los autores.}

Las universidades actualmente se conciben como un instrumento de progreso social, ellas tienen un gran compromiso con la sociedad, deben brindar egresados altamente calificados y comprometidos en ayudar a resolver las problemáticas existentes, es por esto que los estudiantes no pueden mirar los problemas desde una sola óptica y mucho menos buscar la mejor solución desde un mismo ángulo.

El desarrollo de una región, la optimización de los procesos, la solución de los problemas, las creaciones de inventos innovadores, la generación de conocimiento científico y tecnológico no surgen con los conocimientos de una sola disciplina. Como dice Jurjo Torres (2015, citado por Llano Arana, 2016, p. 325) "cuanto mayor bagaje cultural procedente de otras disciplinas podamos poner en interacción, más amplitud alcanzaremos en las miradas".

Acosta, J (2016 p. 216), nos explica lo que pasaría si miramos las cosas desde un solo eje "De no ser así los resultados del proceso de producción de conocimiento se verían influenciados por las deformaciones que impone la selectividad de las perspectivas de análisis a las que se recurre". 
Estamos en un mundo de continuos cambios, que ha evolucionado tecnológicamente a pasos agigantados, el impacto de las TIC e Internet, entre otros, hacen necesario un ambiente educativo diferente, a la vanguardia de las tendencias educativas y tecnológicas. Es así como han surgido nuevas propuestas que dan solución o se han adaptado a las necesidades de algunas personas, como es el caso de la educación virtual o de las herramientas tecnológicas que mejoran los entornos y ambientes de aprendizajes; lo anterior ha permitido que surjan nuevas relaciones entre docentes y estudiantes, a la vez que también han surgido grupos investigativos interdisciplinarios, lo cual ha permitido que los problemas sean vistos desde diferentes ópticas o puntos de vistas al igual que las soluciones; no basta con buscar una solución, sino que esta sea la más adecuada para el tipo de necesidad que se tiene.

En estos tiempos modernos se puede contar con el apoyo de las tecnologías para realizar procesos más fáciles de transdisciplinariedad, Ferreira, A. C (2019 p. 36) nos dice:

"El auge de la tecnología, la simplificación de ésta y su implementación en estudios de analíticas del aprendizaje, permite no solo la integración de otros campos de investigación, sino desarrollar un verdadero enfoque transdisciplinario de las analíticas del aprendizaje, donde la recopilación, el análisis y la interpretación de datos, puede verse desde la perspectiva de otro campo de investigación sin dejar de lado los objetivos".

Y si se mira la solución desde enfoques transdisciplinares, su evaluación también debe ser vista desde esa misma mirada. Peche Cieza, W. (2019 p. 12) nos expresa que:

"Un modelo transdisciplinar de evaluación es aquel donde se da la combinación de diferentes cursos en la que se produce un complejo proceso de integración difusa, a partir de la fusión de más de dos disciplinas, y su resultante final corresponde generalmente a la solución de un problema de investigación. El resultado es un híbrido muy complejo que generalmente incluye elementos multidisciplinarios e interdisciplinarios en su formación".

Por tanto, las universidades deben buscar modelos propios de enseñanza - aprendizaje basados en grupos interdisciplinarios que conlleven a una transdisciplinariedad en la educación. Como dice Sánchez J.R. (2019 p. 82) "hoy en día la transdisciplinariedad se asoma como una alternativa que parece avanzar hacia una verdadera integración del saber humano". 
La toma de conciencia de esta situación implica algo mucho más que una interdisciplinariedad, implica una auténtica transdisciplinariedad, donde las distintas disciplinas están relacionadas unas con otras y transcienden, en cuanto al resultado final es una cualidad emergente, superior a la suma de sus partes.

Trabajar desde estos enfoques implica que las universidades deben reconstruir sus territorios, trabajar más el humanismo y la diversidad, lo cual le abrirá espacios educativos al dialogo de saberes.

\section{REFLEXIONES FINALES}

AlreflexionarsobreINVESTIGACIÓNINTERDISCIPLINARIAYTRANSDISCIPLINARIA COMO TENDENCIA EMERGENTE DE LO SISTÉMICO COMPLEJO DESDE EL PENSAMIENTO CRÍTICO y partiendo desde una perspectiva de pensamiento complejo que se relaciona estrechamente dentro de una pedagogía activa en la cual nos movemos a diario, se observa que ningún evento de las ciencias tiene carácter aislado o desvinculado, sino, que hace parte de un sistema complejo que se relaciona de manera endógena con otros objetos componentes del sistema y de manera exógena con elementos del ambiente, del entorno o del contexto del sistema.

La acción interdisciplinaria la hemos visto de manera patente en cada una de las actividades con las cuales interactuamos a diario en nuestro entorno académico e inclusive en los ambientes ciberhumanista de aprendizaje donde confluyen varias áreas del conocimiento que enriquecen el espectro del estudio de las ciencias de la educación.

Lo transdisciplinario rompe las barreras de lo cognoscente y nos lleva a una visión mucho más amplia del conocimiento tomando como herramienta el desarrollo del pensamiento complejo en el marco de una visión más crítica de nuestro entorno social y científico.

A grandes rasgos, esta tendencia lo que intenta es integrar el enfoque global y complejo del mundo con una formación educacional que tome en cuenta esta visión. Así, todo conocimiento humano que nos permita tomar conciencia de que somos tan sólo una parte componente de un sistema general complejo, será bien recibida para formar parte de la educación.

En esta tendencia se despierta un gran interés por todo lo novedoso de las ciencias y las humanidades. Sin embargo, esto no es una tarea sencilla pues implica derribar los esquemas mentales que, desde el comienzo de la ciencia contemporánea, nos han sido impuestos como es la 
visión de un mundo fragmentario y mecánico. Por ello, en esta tendencia se habla, no sólo de lo que puede ser una nueva educación, sino de una revolución del pensamiento.

La Instituciones Educativas de educación superior tienen el reto de formar individuos capaces de generar conocimiento con pensamiento crítico en una cultura interdisciplinaria y transdisciplinaria que ayude a mejorar las problemáticas sociales con soluciones integras, innovadoras y eficaces.

\section{REFERENCIAS BIBLIOGRÁFÍCAS}

- Acosta, J. (2016). Interdisciplinariedad y transdisciplinariedad: perspectivas para la concepción de la universidad por venir. Alteridad, 11(1), 148-156.

- Bermeo, F., Hernandez J. S., y Tobon S. (2016). Análisis documental de V heurística mediante la cartografía conceptual. Ra Ximhai, 12(6). Recuperado de www.redalyc.org/ pdf/461/46148194006.pdf

- Corrin, L., Alhadad, S., Jones, H. y Colvin, C. (2018). Where is the field of learning analytics heading? Reflections from the Learning Analytics \& Knowledge Conference. Recuperado de: https://bit.ly/2V3pkKR

- Ferreira, A. C., Altamirano, M., Ortega, M. D. L. Á. L., \& González, O. A. G. (2019). Analítica del aprendizaje y las neurociencias educativas: nuevos retos en la integración tecnológica. Revista Iberoamericana de Educación, 80(1), 31-54.

- First World Congress of Transdisciplinarity. (1994). Charter of Transdisciplinarity. Portugal.

- Flinterman, J.; Teclemariam-Mesbah, R. \& Broerse JEW. (2001). Transdisciplinary: th new challenge for biomedical research. Bull Sci, 21:253-66.

- Flinterman, J.; Teclemariam-Mesbah, R. \& Broerse JEW. (2001). Transdisciplinary: the new challenge for biomedical research. Bull Sci, 21:253-66.

- Gómez, C. J., Miralles, P. y Molina, S. (2015). Presentación evaluación, competencias históricas y educación ciudadana. Revista de Estudios Sociales, 52, 9-13. https://doi. org/10.7440/res52.2015.01

- González Velazco, J. M., Carmona Alvarado, F., Arboleda López, A., Porto Solano, A., González Amarillo, Á. M., Avella Ibañez, C. P., ... \& Rúa Ascar, J. M. (2018). Educación transdisciplinar, tecno-didáctica y paradigmas emergentes: para una ciudadanía planetaria.

- Henao Villa, César Felipe, \& García Arango, David Alberto, \& Aguirre Mesa, Elkin Darío, \& González García, Arturo, \& Bracho Aconcha, Rosa, \& Solorzano Movilla, Jose Gregorio, \& Arboleda Lopez, Adriana Patricia (2017). Multidisciplinariedad, interdisciplinariedad y transdisciplinariedad en la formación para la investigación en 
ingeniería. Revista Lasallista de Investigación, 14(1), undefined-undefined. [fecha de Consulta 28 de Septiembre de 2019]. ISSN: 1794-4449. Disponible en: https://www. redalyc.org/articulo.oa? $\mathrm{id}=695 / 69551301017$

- Llano Arana, Lizgrace, Gutiérrez Escobar, Miriam, Stable Rodríguez, Addys, Núñez Martínez, María, Masó Rivero, Rosa, \& Rojas Rivero, Bárbara. (2016). La interdisciplinariedad: una necesidad contemporánea para favorecer el proceso de enseñanza aprendizaje. MediSur, 14(3), 320-327. Recuperado en 24 de septiembre de 2019, de http://scielo.sld.cu/scielo.php?script=sci_arttext\&pid=S1727-897X201600030 0015\&lng=es\&tlng=es

- Lodge, J., Hoovarth, J. y Corrin, L. (ed.) (2018) Learning Analytics in the Classroom: ranslating Learning Analytics Research for Teachers. (1st Edition). London: Taylor and Francis. https://doi.org/10.4324/9781351113038

- López, N., Lugo, M.T., Toranzos, L. (2014). Informe sobre tendenciassociales y educativasen América Latina2014: Políticas TIC en los sistemaseducativosde América Latina. Recuperado de: http://repositorio.minedu.gob.pe/handle/123456789/3455

- Malaina, Á. (2016). La movilidad juvenil europea y el espacio transnacional emergente: una aproximación desde las teorías de la complejidad. Revista de Estudios de Juventud, (113), 53-65.

- Martínez Villalba, J. A., \& Sánchez Muñoz, S. (2018). Generación de competencias con base en la gestión de conocimiento científico. REICE. Revista Iberoamericana sobre Calidad, Eficacia y Cambio en Educación.

- Mejía, Y. A. C. (2017). Transdisciplinariedad y transversalidad. Revista Publicando, 4(11 (1)), 499-512.

- Morin, Edgar (1999a). Introduction á une politique de 1 'homme. París: Editions du Seuil. Morin, Edgar (1999b). Los 7 saberes necesarios para la educación del futuro. París: UNESCO.

- Morin, Edgar (2001). La cabeza bien puesta. Repensar la reforma. Repensar el pensamiento. Barcelona: Seix Barral.

- Morin, Edgar (2004). Pour entrer dans le XXI e siécle. París: Editiones du Seuil

- Morín, Edgar (2005). Complexité restreinte, complexité générale. Coloquio Intelligence de la Complexite': e'piste'mologie etpragmatique, Cerisy La Salle, 26 de junio, 2005.

- Morín, Edgar (2013). Mes philosophes. París: Pluriel. Morín, Edgar (2015a). L’aventure de La Méthode. Paris: Seuil.

- Morín, Edgar (2015b). Penser global. París: Champs / Essais. 
- Morín, Edgar (2016). Écologiser l'homme. Paris: LemiuX éditeur.

- Morín, Edgar \& Kareh Tager, Djénane (2010). Mi camino. La vida y la obra del padre del pensamiento complejo. Barcelona: Gedisa.

- Morin, Edgar \& Le Moigne, Jean—Louis (1999). L 'intelligence de la complexité. Paris: L'Harmattan.

- Ortiz, M., Vicedo, A. y García, J. (2016). Competencias, ética y valores en la formación del especialista de Pediatría. Revista Habanera de Ciencias Médicas, 15(2), 247-258.

- Peche Cieza, W. (2019). Modelo transdisciplinar de evaluación para mejorar la calidad del proceso de enseñanza aprendizaje de los estudiantes del programa de licenciatura en educación modalidad mixta, especialidad de Educación Inicial-sede Ferreñafe de la Universidad Nacional Pedro Ruiz Gallo de Lambayeque.

- Pereira, H. (2011). Implementación de la gestión del conocimiento en la empresa. Éxito Empresarial, 135, 1-6.

- Peres-Cajías, G. (2019). METOdología PARA UN ACERCAMIENTO POSTMODERNO HACIA LAS COMUNIDADES DE APRENDIZAJE TRANSDISCIPLINARIAS. Punto Cero, 24(38), 45-56.

- Pinto Molina, María. (1989). Introducción al análisis documental y sus niveles: el análisis de contenido. Boletín de la ANABAD, 39(2), 323-342. Recuperado de (file:///C:/Users/ Nelly/Downloads/Dialnet-IntroduccionAlAnalisisDocumentalYSusNiveles-798857.pdf)

- Rodríguez Zoya, Leonardo 8: Roggero, Pascal (2015, septiembre—diciembre). Modelos basados en agentes: aportes epistemológicos y teóricos para la investigación social. Revista Mexicana de Ciencias Políticas y Sociales, Año LX, 60(225), 227-261. Universidad Nacional Autónoma de México / Nueva Época

- Rodríguez Zoya, Leonardo \& Rodríguez Zoya, Paula (2014, diciembre). El espacio controversial de los sistemas complejos. Estudios de filosofia, No.50, 103-129. Universidad de Antioquia.

- Roggero, Pascal (2013). Para una sociología según El método. En Esteban Ruiz Ballesteros \& José Luis Solana Ruiz (Eds), Complejidady ciencias sociales. Sevilla: Universidad Internacional de Andalucía.

- Romero, C. A. (2007). Gestión del conocimiento, asesoramiento y mejora escolar. El caso de la escalera vacía. Profesorado. Revista de Currículum y Formación del Profesorado, 11(1), 1-28.

- Sánchez, J. R. A., Alvarado, G. P., \& Santoyo, A. A. (2019). Herramientas metodológicas para la investigación transdisciplinaria en las ciencias sociales. Transdisciplinariedad y 
Investigación interdisciplinaria y transdisciplinaria como tendencia emergente de lo sistémico complejo desde el pensamiento crítico

Daniel A. González O.; Luis A. Padilla D.; Nelly M. Zúñiga D. (63:83)

Desarrollo del Conocimiento en las Humanidades y Ciencias Sociales, 80 .

- Solorzano Movilla, J. G., Henao Villa, C. F., Bracho Aconcha, R., Arboleda Lopez, A. P., García Arango, D. A., Aguirre Mesa, E. D., \& González García, A. (2017). Multidisciplinariedad, interdisciplinariedad y transdisciplinariedad en la formación para la investigación en ingeniería.

- Torres Santomé J. Asignaturas enfrentadas en busca de espacio. Cuadernos de Pedagogía. $2015 ; 456: 8$ 